\title{
Index of Volume 197 (1991)
}

\section{Number 1/2}

W. Schwieger, K.-H. Bergk, D. Heidemann, G. Lagaly, K. Beneke

Hochauflösende ${ }^{29}$ Si-Festkörper-NMR-Untersuchungen am synthetischen Natriumsilicathydrat Makatit und dessen kristalliner Kieselsäure

W. A. Dollase, C. R. Ross II

Crystal structure of orientationally disordered $\mathrm{Na}_{2}(\mathrm{Ca}, \mathrm{Sr}) \mathrm{SiO}_{4} \ldots$. .

H. Effenberger, H. Fuess, G. Müller, T. Vogt

Crystal structure and hydrogen bonding in $\mathrm{Li} / \mathrm{H}$-exchanged petalite, $\mathrm{HAlSi}_{4} \mathrm{O}_{10} \ldots \ldots \ldots \ldots \ldots . \ldots . \ldots . . . \ldots$

V. Busetti, G. Cevasco, G. Leandri

Crystal structure of diaryl sulphur diimides. Ortho-substituted derivatives

A. De, G. Biswas

Crystal structure of a substituted pyranocarbazole alkaloid $\mathrm{N}$-allyl girinimbine

L. S. Dent Glasser, R. P. Gunawardane, R. A. Howie

The crystal structure of sodium strontium silicate, $\mathrm{Na}_{4} \mathrm{SrSi}_{3} \mathrm{O}_{9} \ldots$. .

K. H. Lii, C. H. Li, T. M. Chen, S. L. Wang

Synthesis and structural characterization of sodium vanadyl(IV) orthophosphate $\mathrm{NaVOPO}_{4} \ldots \ldots . \ldots . . . \ldots$

Y. Ito, S. Kuehner, S. Ghose

Phase transitions in leucite determined by high temperature, single crystal $\mathrm{X}$-ray diffraction

A. J. C. Wilson

Space groups rare for molecular organic structures: The arithmetic crystal class $m m m P$

G. Paiaro, L. Pandolfo, P. Ganis, G. Valle

The crystal structure of $\mathrm{Pt}(\mathrm{II})$-trans-[(bis-tricyclohexylphosphine)-( $\eta^{1}-h y$ dro-formyl-ter-butylamido)(hydride)]

Gravegliaite, $\mathrm{MnSO}_{3} \cdot 3 \mathrm{H}_{2} \mathrm{O}$, a new mineral from Val Graveglia (Northern Apennines, Italy) 
M. Rieder, Z. Weiss

Oblique-texture X-ray photographs: more information from powder diffraction

U. Dettlaff-Weglikowska, E. Hey-Hawkins, D. Thiery, H. G. von Schnering Struktur von Tetraphenylphosphonium-[(Brenzkatechin)chlorid]

J. K. Cockcroft, A. N. Fitch

The structure of solid dichlorodifluoromethane $\mathrm{CF}_{2} \mathrm{Cl}_{2}$ by powder neutron diffraction

\section{Short Communication}

R. Dronskowski, A. Simon

Verfeinerung der Kristallstruktur von $\mathrm{Mo}_{5} \mathrm{Ge}_{3} \ldots \ldots$. . . .

\section{New Crystal Structures}

\section{B. Eisenmann, A. Hofmann}

Crystal structure of hexasodium di- $\mu$-tellurido-bis(ditelluridoaluminate),

$\mathrm{Na}_{6} \mathrm{Al}_{2} \mathrm{Te}_{6}$

B. Eisenmann, A. Hofmann

Crystal structure of hexasodium di- $\mu$-selenido-bis(diselenidoaluminate), $\mathrm{Na}_{6} \mathrm{Al}_{2} \mathrm{Se}_{6}$

B. Eisenmann, A. Hofmann

Crystal structure of hexasodium di- $\mu$-thio-bis(dithiogallate) $-\mathrm{I}$, $\mathrm{Na}_{6} \mathrm{Ga}_{2} \mathrm{~S}_{6}$

B. Eisenmann, A. Hofmann

Crystal structure of hexapotassium di- $\mu$-tellurido-bis(ditelluridogallate, $\mathrm{K}_{6} \mathrm{Ga}_{2} \mathrm{Te}_{6} \ldots \ldots \ldots \ldots \ldots \ldots$

B. Eisenmann, A. Hofmann

Crystal structure of hexasodium di- $\mu$-thio-bis(dithiogallate) - II, $\mathrm{Na}_{6} \mathrm{Ga}_{2} \mathrm{~S}_{6}$. . . . . . . . . . . . . . . . . . . . . . . . .

B. Eisenmann, A. Hofmann

Crystal structure of hexasodium di- $\mu$-selenido-bis(diselenidogallate), $\mathrm{Na}_{6} \mathrm{Ga}_{2} \mathrm{Se}_{6}$

B. Eisenmann, A. Hofmann

Crystal structure of hexasodium di- $\mu$-thio-bis(dithioindate), $\mathrm{Na}_{6} \operatorname{In}_{2} \mathrm{~S}_{6}$

B. Eisenmann, A. Hofmann

Crystal structure of hexapotassium di- $\mu$-selenido-bis(diselenidogallate), $\mathrm{K}_{6} \mathrm{Ga}_{2} \mathrm{Se}_{6} \ldots \ldots \ldots \ldots \ldots \ldots$

B. Eisenmann, A. Hofmann

Crystal structure of hexapotassium di- $\mu$-thio-bis(dithiogallate), $\mathrm{K}_{6} \mathrm{Ga}_{2} \mathrm{~S}_{6}$ 
B. Eisenmann, A. Hofmann

Crystal structure of hexapotassium di- $\mu$-tellurido-bis(ditelluridoaluminate), $\mathrm{K}_{6} \mathrm{Al}_{2} \mathrm{Te}_{6}$

B. Eisenmann, A. Hofmann

Crystal structure of heptasodium catenaoctaselenidotriindate(III), $\mathrm{Na}_{7} \mathrm{In}_{3} \mathrm{Se}_{8}$

B. Eisenmann, A. Hofmann

Crystal structure of hexasodium di- $\mu$-thio-bis(dithioaluminate) $-\mathrm{HT}$, $\mathrm{Na}_{6} \mathrm{Al}_{2} \mathrm{~S}_{6}$. . . . . . . . . . . . . . . . . . . .

B. Eisenmann, A. Hofmann

Crystal structure of pentapotassium tetraselenidogallate(III), $\mathrm{K}_{5} \mathrm{GaSe}_{4}$

B. Eisenmann, A. Hofmann

Crystal structure of a calcium thioindate, $\mathrm{Ca}_{0.76} \mathrm{In}_{2.84} \mathrm{~S}_{5}$. . . . . . .

B. Eisenmann, A. Hofmann

Crystal structure of strontium phyllotetrathiodiindate(III), $\operatorname{SrIn}_{2} \mathrm{~S}_{4}$.

B. Eisenmann, A. Hofmann

Crystal structure of pentasodium tetrathioindate(III), $\mathrm{Na}_{5} \mathrm{InS}_{4} \ldots$. .

B. Eisenmann, A. Hofmann

Crystal structure of sodium phyllo-diselenidoaluminate, $\mathrm{NaAlSe}_{2}$. .

B. Eisenmann, A. Hofmann

Crystal structure of hexapotassium di- $\mu$-selenido-bis(diselenidoaluminate), $\mathrm{K}_{6} \mathrm{Al}_{2} \mathrm{Se}_{6}$

\section{Number 3/4}

Th. Kellersohn, B. Engelen, H. D. Lutz, H. Bartl, B. P. Schweiss, H. Fuess Local pseudosymmetry of the water molecule in $\mathrm{BaBr}_{2} \cdot 2 \mathrm{H}_{2} \mathrm{O}$, a neutron diffraction study

Su Genbo, Li Zhengdong

Crystal growth and physical properties of disodium sulfosalicylate trihydrate

J. Novotný, J. Ondráček, B. Kratochvíl, K. Čapek

The crystal and molecular structure of 2,3'-anhydrosucrose . . . . .

Fan Hai-fu, H. Quan, M. M. Woolfson

Proteins and direct methods

A. Mostad, S. Natarajan

Crystal and molecular structure of the pyroelectric phase of TGS at $150 \mathrm{~K}$ 


\section{E. Papavinasam}

Crystal structure of [(bis(glycinato) copper(II)dibromide)((bis(glycine) dilithium(I)) dihydrate $)]$. $\left[\left(\left(\mathrm{C}_{2} \mathrm{H}_{4} \mathrm{NO}_{2}\right)_{2} \mathrm{CuBr}_{2}\right) \cdot\left(\left(\mathrm{C}_{2} \mathrm{H}_{5} \mathrm{NO}_{2}\right)_{2} \mathrm{Li}_{2}\right) \cdot 2\right.$ $\mathrm{H}_{2} \mathrm{O}$. . . . . . . . . . . . . . . . .

L. Born, G. Heywang

The crystal structure of the radical anion salt of naphthalene tetracarboxylic dianhydride (NTDA) and 5,6-dihydro-4a,6a-phenanthrolene dionium (DPD): a new charge transfer complex .

L. Eriksson, P. Wang, P.-E. Werner

Crystal structure and phase transition of $\alpha-\mathrm{Cu}_{2} \mathrm{Hgl}_{4} \ldots \ldots$. . . .

\section{New Crystal Structures}

M. Jansen, G. Q. Wu, K. Königstein

Crystal structure of caesium ytterbium diphosphate, $\mathrm{CsYbP}_{2} \mathrm{O}_{7}$

B. Lüer, M. Jansen

Crystal structure refinement of tetraphosphorous nonaoxide, $\mathrm{P}_{4} \mathrm{O}_{9}$.

M. Somer, K. Peters, H. G. von Schnering

Crystal structure of caesium potassium rubidium 2,5-diantimonido-tristiba-2,5-digalla-cyclopentanate, $\mathrm{Cs}_{3.31} \mathrm{~K}_{2.25} \mathrm{Rb}_{1.44} \mathrm{Ga}_{2} \mathrm{Sb}_{5}$

B. Eisenmann, J. Jäger

Crystal structure of caesium catena-tritelluroaluminate, $\mathrm{CsAlTe}_{3}$. .

B. Eisenmann, J. Jäger

Crystal structure of hexacaesium di- $\mu$-telluro-bis(ditelluroaluminate), $\mathrm{Cs}_{6} \mathrm{Al}_{2} \mathrm{Te}_{6} \ldots \ldots \ldots \ldots \ldots \ldots$

B. Eisenmann, R. Zagler

Crystal structure of tripotassium tritelluroantimonate(III), $\mathrm{K}_{3} \mathrm{SbTe}_{3}$.

B. Eisenmann, R. Zagler

Crystal structure of tripotassium tritellurobismutate(III), $\mathrm{K}_{3} \mathrm{BiTe}_{3}$. . 257

B. Eisenmann, J. Jäger, R. Zagler

Crystal structure of tetrapotassium tetratellurodiarsenate(II), $\mathrm{K}_{4} \mathrm{As}_{2} \mathrm{Te}_{4}$

B. Eisenmann, R. Zagler

Crystal structure of tetrasodium tetratellurodiarsenate(II), $\mathrm{Na}_{4} \mathrm{As}_{2} \mathrm{Te}_{4}$

B. Eisenmann, J. Klein, M. Somer

Crystal structure of pentarubidium triphosphidogermanate, $\mathrm{Rb}_{5} \mathrm{GeP}_{3}$

B. Eisenmann, J. Klein, M. Somer

Crystal structure of decasodium di- $\mu$-arsenido-bis(diarsenidogermanate),

$\mathrm{Na}_{10} \mathrm{Ge}_{2} \mathrm{As}_{6}$

B. Eisenmann, J. Klein, M. Somer

Crystal structure of decasodium di- $\mu$-arsenido-bis(diarsenidosilicate),

$\mathrm{Na}_{10} \mathrm{Si}_{2} \mathrm{As}_{6} \ldots \ldots \ldots \ldots . . \ldots$ 
B. Eisenmann, J. Klein, M. Somer

Crystal structure of decasodium di- $\mu$-phosphido-bis(diphosphidostannate(IV)), $\mathrm{Na}_{10} \mathrm{Sn}_{2} \mathrm{P}_{6}$

B. Eisenmann, J. Klein, M. Somer

Crystal structure of tetrapotassium diarsenidocadmate, $\mathrm{K}_{4} \mathrm{CdAs}_{2}$. . . 271

B. Eisenmann, J. Klein, M. Somer

Crystal structure of pentarubidium triarsenidosilicate, $\mathrm{Rb}_{5} \mathrm{SiAs}_{3} \ldots 273$

B. Eisenmann, J. Klein, M. Somer

Crystal structure of trisodium dipotassium triphosphidosilicate, $\mathrm{Na}_{3} \mathrm{~K}_{2} \mathrm{SiP}_{3} \ldots \ldots \ldots \ldots . \ldots . \ldots 275$

B. Eisenmann, J. Klein, M. Somer

Crystal structure of dipotassium catena-phosphidoaurate(I), $\mathrm{K}_{2} \mathrm{AuP}$.

B. Eisenmann, J. Klein

Crystal structure of tetrasodium monopotassium triarsenidogermanate,

$\mathrm{Na}_{4} \mathrm{KGeAs}_{3}$. . . . . . . . . . . . . . . . . . . 279

G. Cordier, H. Ochmann

Crystal structure of disodium phyllo-triantimonidodiindate, $\mathrm{Na}_{2} \mathrm{In}_{2} \mathrm{Sb}_{3}$

G. Cordier, H. Ochmann

Crystal structure of disodium phyllo-triarsenidodialuminate, $\mathrm{Na}_{2} \mathrm{Al}_{2} \mathrm{As}_{3} \ldots \ldots \ldots \ldots . \ldots . . \ldots$

G. Cordier, H. Ochmann

Crystal structure of disodium phyllo-triarsenidodigallate, $\mathrm{Na}_{2} \mathrm{Ga}_{2} \mathrm{As}_{3}$

G. Cordier, H. Ochmann

Crystal structure of dipotassium phyllo-triarsenidodigallate, $\mathrm{K}_{2} \mathrm{Ga}_{2} \mathrm{As}_{3}$

G. Cordier, H. Ochmann

Crystal structure of dipotassium phyllo-triantimonidodigallate, $\mathrm{K}_{2} \mathrm{Ga}_{2} \mathrm{Sb}_{3}$

G. Cordier, H. Ochmann

Crystal structure of dipotassium phyllo-triantimonidodiindate, $\mathrm{K}_{2} \mathrm{In}_{2} \mathrm{Sb}_{3} \ldots \ldots \ldots \ldots \ldots \ldots \ldots$

G. Cordier, H. Ochmann

Crystal structure of dipotassium phyllo-triarsenidodiindate, $\mathrm{K}_{2} \mathrm{In}_{2} \mathrm{As}_{3}$

G. Cordier, H. Ochmann

Crystal structure of tripotassium phyllo-triarsenidoindate, $\mathrm{K}_{\mathbf{3}} \mathrm{In}_{2} \mathrm{As}_{3}$

G. Cordier, H. Ochmann

Crystal structure of potassium phyllo-diantimonidogallate, $\mathrm{KGaSb}_{2}$.

M. Nieger, O. Altmeyer, E. Niecke

Crystal structure of 1,2-bis-[tris-(trimethylsilyl)hydrazino]-1,2-dibromodiphosphane, $\left(\left(\left(\mathrm{CH}_{3}\right)_{3} \mathrm{Si}\right)_{3} \mathrm{~N}_{2}(\mathrm{Br}) \mathrm{P}\right)_{2}$ 


\section{F. Knoch}

Crystal structure of 8-(2-hydroxyethoxy)quinoline dihydrate, $\mathrm{C}_{9} \mathrm{H}_{6} \mathrm{NOCH}_{2} \mathrm{CH}_{2} \mathrm{OH}\left(\mathrm{H}_{2} \mathrm{O}\right)_{2} \ldots \ldots \ldots$. . . . . . . . .

W. P. Bosman, P. T. Beurskens, G. Admiraal, R. P. Sijbesma, R. J. M. Nolte

Crystal structure of 1,6:3,4-bis(4,5-benzo-3,6-dimethoxy-1,2-xylylene) tetrahydro-3a,6a-diphenylimidazo[4,5-d]imidazole-2,5(1H,3H)-dione, $\mathrm{C}_{44} \mathrm{H}_{38} \mathrm{~N}_{4} \mathrm{O}_{6}$

K. Peters, E.-M. Peters, H. G. von Schnering, W. Adam, S. E. Bottle Crystal structure of 2,4,4-triphenyl-2-aza-3-oxabicyclo[3.3.0]oct-5-ene, $\mathrm{C}_{24} \mathrm{H}_{21} \mathrm{NO} \ldots \ldots \ldots \ldots$. . . . . . . . . . 30

G. Cordier, C. Röhr

Crystal structure of strontium gold gallium (1/2/2), $\mathrm{SrAu}_{2} \mathrm{Ga}_{2} \ldots$. . 31?

G. Cordier, C. Röhr

Crystal structure of calcium copper indium (1/4/1), $\mathrm{CaCu}_{4}$ In . . . . 314

Book Reviews

W. Borchardt-Ott

Kristallographie. 3. Auflage. (Rez.: J. Bohm) . . . . . . . . . . 317

R. Vanselow, R. Howe (Eds.)

Chemistry and Physics of Solid Surfaces VIII. (Rez.: A. Meisel) . . . 313

R. Iffländer

Festkörperlaser zur Materialbearbeitung. (Rez.: W. Pompe) . . . . 318

A. R. Hölzel

Systematics of Minerals. (Rez.: H.-J. Höbler) . . . . . . . . . . . 319

H. Zabel, S. A. Solin (Eds.)

Graphite Intercalation Compounds I. (Rez.: H. Eschrig) . . . . . . . 320

A. Lösche

Molekulare Ordnung und Orientierung, insbesondere bei Flüssigkristal-

len. (Rez.: D. Demus) . . . . . . . . . . . . . . . . 321

C. B. Harris, E. P. Ippen, G. A. Mourou, A. H. Zewail (Eds.)

Ultrafast Phenomena VII. (Rez.: K. Kreher) . . . . . . . . . . 322

Author Index of Volume $197 \quad 323$

Subject Index of Volume 197

Formulae Index of Volume 197 